\title{
DISTRIBUIÇÃO DE Stygarctus bradypus SCHULZ, 1951 (TARDIGRADA, HETEROTARDIGRADA) NA MARGEM SUL DA ILHA DE ITAMARACÁ (PE, BRASIL)
}

\author{
MÔNICA MARINHO VERÇOSA ${ }^{1.3}$, ÉRIKA CAVALCANTE LEITE DOS SANTOS ${ }^{1}$, JOSÉ ROBERTO B. DE SOUZA ${ }^{2}$ \& CLÉLIA M. C. DA \\ $\mathrm{ROCHA}^{1}$ \\ ${ }^{1}$ Universidade Federal Rural de Pernambuco, Depto. de Biologia. Av. Manoel de Medeiros, s/n. Dois Irmãos. Recife - PE, Cep: $52171-900$ - \\ Brasil.). ${ }^{2}$ Universidade Federal de Pernambuco, Centro de Ciências Biológicas, Depto. Zoologia. Av. Prof. Nelson Chaves, s/n, Cidade \\ Universitária. Recife - PE, Cep: 50739-000 -, Brasil. ${ }^{3}$ monicamvercosa@yahoo.com.br
}

\begin{abstract}
Este estudo objetivou investigar a distribuição espacial e temporal de Stygarctus bradypus Schulz, 1951 (Tardigrada, Heterotardigrada) em uma praia arenosa na margem sul da ilha de Itamaracá, $40 \mathrm{~km}$ a norte do Recife (PE, Brasil). As amostras foram coletadas em dois períodos do ano caracterizados por estiagem e chuva, através de duas transversais na região entre-marés, durante a baixa-mar. Foram identificados 337 indivíduos pertencentes à espécie Stygarctus bradypus. Houve diferenças significativas nas populações de $S$. bradypus dos dois transectos e entre os andares da praia, mas não entre os estratos sedimentares nem entre os períodos do ano. Concluiu-se que a espécie não tem a sua distribuição influenciada pelo tamanho dos grãos dos sedimentos nem pela presença de águas intersticiais, preferencialmente colonizando o médiolitoral superior da praia estudada, concentrando-se entre $10 \mathrm{~cm}$ a $20 \mathrm{~cm}$ de profundidade no sedimento ao longo do ano.
\end{abstract}

RESUMO

PALAVRAS CHAVE: meiofauna, praia arenosa, Itamaracá, Stygarctus bradypus, Tardigrada.

\section{ABSTRACT}

Distribution of Stygarctus bradypus Schulz, 1951 (Tardigrada, Heterotardigrada) at the southern edge of Itamaracá Island (PE, Brazil).

This work aimed to investigate the spatial and temporal distribution of Stygarctus bradypus Schulz, 1951 (Tardigrada, Heterotardigrada) in a sandy beach in the southern edge of Itamaracá Island, $40 \mathrm{~km}$ north far from Recife (PE, Brazil). Samples were taken in two different periods of the year characterized by dryness and rain, along two coastal transects through the intertidal zone, during low tides. One identified 337 specimens of Stygarctus bradypus. There was a significant difference between the populations of $S$. bradypus from the two transects and between the steps of the beach, but not between the sedimentary layers and between the two periods of the year. It was concluded that this species does not have its distribution influenced by the sediments granulometry nor for the presence of interstitial water, preferentially colonizing the upper mid-littoral of the studied beach, concentrating its population between $10 \mathrm{~cm}$ and $20 \mathrm{~cm}$ depth in the sediments throughout the whole year.

KEY WORDS: meiofauna, sandy beach, Itamaracá, Stygarctus bradypus, Tardigrada.

\section{INTRODUÇÃO}

A distribuição espacial da meiofauna em praias arenosas depende principalmente das características do sedimento. Em regiões entre-marés com areia grossa os organismos podem penetrar até $1 \mathrm{~m}$ no sedimento (Souza-Santos et al. 2004). Por outro lado, em ambientes onde os grãos sedimentares são menores, a meiofauna fica restrita aos primeiros centímetros superficiais, devido à oclusão no espaço intersticial e redução da quantidade de oxigênio disponível nas camadas mais profundas (Fleeger \& Decho 1987). A distribuição horizontal é afetada principalmente pela ação das marés (McLachlan 1983). Além dos fatores abióticos, os biológicos também exercem influência na meiofauna, principalmente a disponibilidade alimentar, que condiciona a distribuição desta fauna em mosaicos (Findlay 1981).

Tardígrados são reconhecidos como componentes permanentes do meiobentos (RenaudDebyser 1956, 1959a), sendo muitas espécies intersticiais em zonas entre-marés ou infralitoral raso de praias arenosas (Kristensen \& Higgins 1984). Segundo Grimaldi De Zio et al. (1983a) em areias da zona entre-marés são encontradas principalmente espécies mesopsâmicas da família Batillipedidae, enquanto na superfície de sedimentos mais grosseiros ocorrem muitos Halechiniscidae e Stygarctidae.

Stygarctidae Schulz, 1951 são Heterotardigrada que têm tipicamente cinco pares de apêndices cefálicos e apenas um cirro mediano. Pernas sem dedos, mas com 4 garras apresentando longos filamentos. São geralmente encontrados na superfície de areias mais grossas (Ramazotti \& Maucci 1983). Stygarctus bradypus Schulz, 1951 mede de 95 a 110 $\mu \mathrm{m}$ de comprimento, e tem ocorrência registrada para o mar do Norte (Schulz 1951, Schmidt 1968, 1969), Golfo de Bengala (Rao \& Ganapati 1968), Estados Unidos (McGinty \& Higgins 1968, Kristensen \& Nehuaus 1999), Itália (de Zio 1965, de Zio Grimaldi \& Gallo D'Addabbo 2001, Grimaldi de Zio et al. 1983b, 
Grimaldi de Zio et al. 1983a, Grimaldi de Zio \& D’Addabbo Gallo 1987, Grimaldi de Zio et al. 1982a, Grimaldi de Zio et al. 1979, Grimaldi de Zio et al. 1980a, Grimaldi de Zio et al. 1980b), França (RenaudDebyser 1956, 1959a, 1963, Hondt 1970), Malásia (Renaud-Mornant \& Serene 1967), Bahamas (RenaudDebyser 1959b, 1963), Jamaica (McGinty \& Higgins 1968) e Índia (Rao \& Ganapati 1968), preferencialmente na região entre-marés de praias arenosas, colonizando também o infralitoral raso até 15 $\mathrm{m}$ de profundidade (Lindgren 1971, De Zio Grimaldi et al. 2003), com relatos de abruptas variações de densidades populacionais (Renaud-Debyser 1956, Schmidt 1969). Não há registros a respeito da bioecologia da espécie no Hemisfério Sul.

Neste estudo foi investigada a presença de $S$. bradypus em duas situações temporais (período seco e período chuvoso) na margem sul da ilha de Itamaracá (PE), sob a hipótese de que a espécie se distribui diferenciadamente ao longo da zona entremarés em função do trecho do litoral. Buscou-se relacionar seu padrão de distribuição a parâmetros ambientais de oxigênio das águas intersticiais, granulometria dos sedimentos e pluviometria da região.

\section{MATERIAL E MÉTODOS}

A ilha de Itamaracá localiza-se cerca de $40 \mathrm{~km}$ a norte do Recife, entre as latitudes $7^{\circ} 34^{\prime} \mathrm{S}$ e $7^{\circ} 55^{\prime} \mathrm{S}$, e longitudes $34^{\circ} 48^{\prime} \mathrm{W}$ e $34^{\circ} 52^{\prime} \mathrm{W}$ (Fig. 1). Nas zonas litorâneas e costeiras da llha encontram-se prados de Halodule wrightii Archers 1886, os quais se estendem na direção Norte-Sul por aproximadamente $12,5 \mathrm{~km}$ (Martins 1997). É separada do continente pelo canal de Santa Cruz, apresentando temperatura média anual de $26{ }^{\circ} \mathrm{C}$ e média pluviométrica de $900 \mathrm{~mm}$ a $2.000 \mathrm{~mm}$. Apresenta clima tropical, quente e úmido do tipo Aws, segundo a classificação do sistema Köppen, com duas estações bem definidas, uma seca, correspondente ao período de setembro a janeiro, e uma chuvosa que corresponde ao período de fevereiro a agosto (Medeiros et al. 2001, Dos Santos et al. 2006). Os ventos predominantes são alísios de sudeste com velocidade media de $3,2 \mathrm{~m} / \mathrm{s}$ a qual tende a aumentar $(4,0 \mathrm{~m} / \mathrm{s})$ em julho (Medeiros et al. 2001). Devido ao aquecimento do continente durante a estação seca, a pressão barométrica diminui, introduzindo um componente ao norte, com ventos soprando do nordeste (Medeiros et al. 2001).

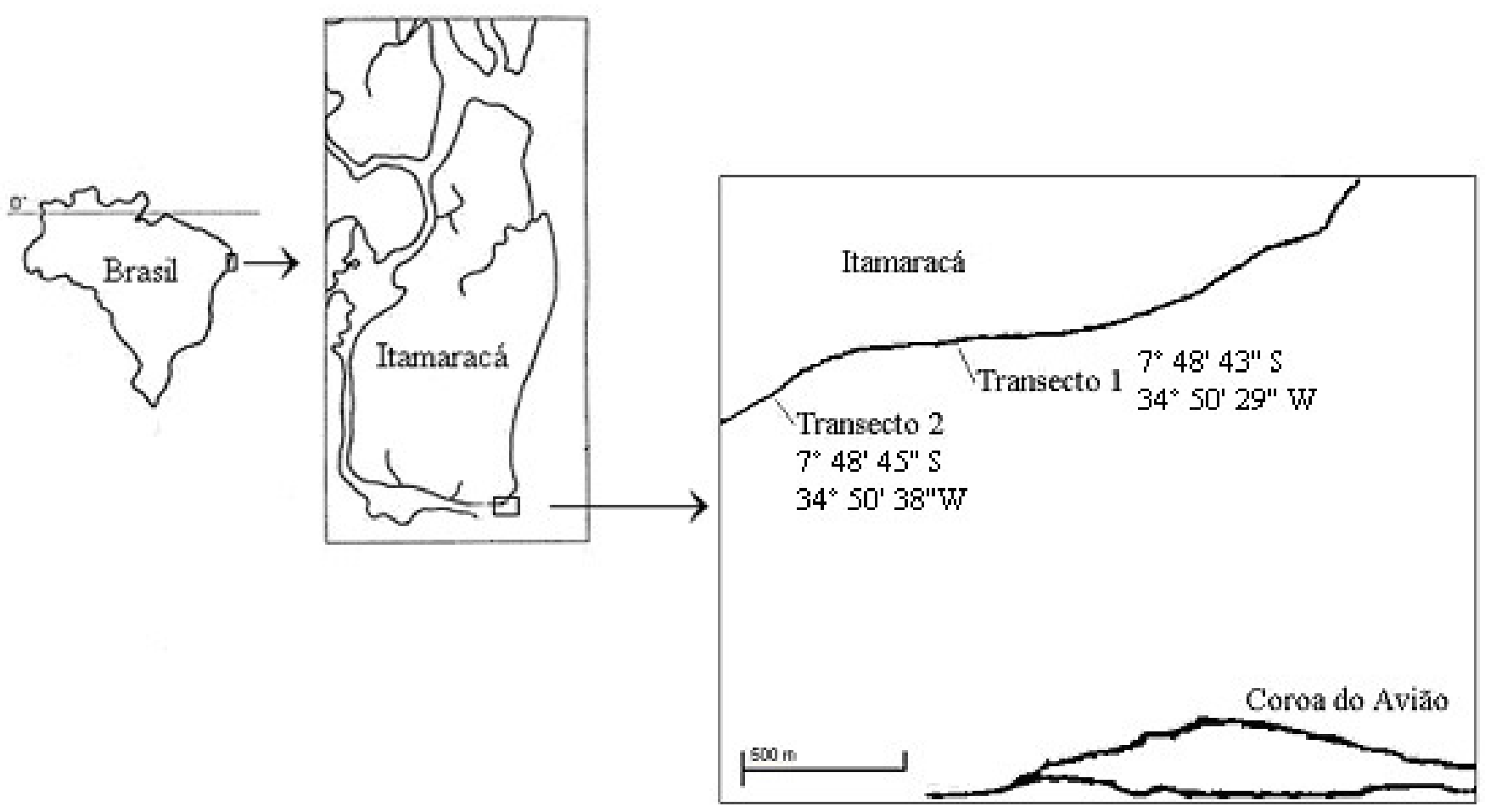

Figura 1 - Localização da área indicando locais de coleta. 
$\mathrm{Na}$ margem sul da ilha de Itamaracá foram realizadas 4 coletas bio-sedimentológicas nos meses de junho, julho (período chuvoso) e novembro de 2005 e janeiro de 2006 (período seco), durante as marés baixas ao longo de dois transectos perpendiculares à linha de praia com distância de cerca de $500 \mathrm{~m}$ entre si. $\mathrm{O}$ transecto 1 está sujeito a uma ação maior das ondas que o transecto 2. Além disso, a direção do vento predominante na área de estudo é quase paralela à linha de costa, possibilitando que o transecto 2 tenha uma maior área para a ação selecionadora do vento que o primeiro.

Em cada transecto foram demarcados 3 pontos de coleta na região entre-marés, sendo um ponto na parte superior, outro na região intermediária e o último na região inferior do médio-litoral. Foram coletadas quatro amostras em cada ponto, sendo três para análises biológicas e uma para análise granulométrica. As amostras foram obtidas através de um testemunhador cilíndrico de PVC (Hulings \& Gray 1971) com $10 \mathrm{~cm}^{2}$ de área. Cada amostra foi dividida em três estratos sedimentares de $10 \mathrm{~cm}$ cada um. Foi feita a aferição dos teores de oxigênio dissolvido da água intersticial através de oxímetro portátil sempre que se apresentava em quantidade suficiente para a leitura direta nos pontos de coleta. Para análises granulométricas foram utilizados $100 \mathrm{~g}$ de cada estrato sedimentar.

A extração da fauna foi feita através de técnicas de rotina para estudos meiofaunísticos (Elmgren 1966). A triagem foi feita numa placa de Dolffus sob estereomicroscópio. A meiofauna foi identificada em níveis taxonômicos altos, sendo os Tardigrada retirados para resolução taxonômica. Os indivíduos foram extraídos manualmente com uma pinça de aço inox no 5 e colocados em solução de $75 \%$ glicerol e $25 \%$ formol a $10 \%$ por 10 minutos. Feito isto foram transferidos para uma gota de glicerol puro em lamínula com círculo de parafina, numa adaptação da técnica descrita por Cobb (1917) (Dos Santos et al. 2006).

A análise da distribuição espacial dos parâmetros granulométricos (diâmetro-médio, mediana, desvio-padrão, curtose, assimetria e grau de seleção) foi processada através do programa estatístico SYSGRAN 3.0, que utiliza os parâmetros de Folk \& Ward (1957).

As densidades populacionais de $S$. bradypus foram expressas através do número de indivíduos por $10 \mathrm{~cm}^{2}$ de sedimento.

Todos os resultados obtidos para análises granulométricas e densidades populacionais de $S$. bradypus foram analisados sob o ponto de vista das diferenças entre as diferentes situações de coleta (transectos, andares de praia, estratos sedimentares, períodos do ano). As relações de $S$. bradypus com os parâmetros ambientais foram testadas através de correlação não-paramétrica de Spearman. Os dados foram transformados à raiz quarta, para se testar a diferença de abundância. Usou-se o teste de Bartlett para verificar a homoscedasticidade dos dados. Estes foram testados através de ANOVA fatorial e teste a posteriori de Tukey, com auxílio do programa STATISTICA 6.0.

\section{RESULTADOS}

Os dois transectos estudados mostraram-se diferentes em muitos aspectos: quanto à presença de água, ao tamanho dos grãos dos sedimentos e quanto à presença de Stygarctus bradypus.

A presença de água intersticial somente ocorreu regularmente nos andares inferiores da praia (médio-litoral e médio-litoral inferior), quando foi medida a concentração de $\mathrm{O}_{2}$. Os valores de oxigênio dissolvido encontrados variaram desde $0,6 \mathrm{mg} / \mathrm{ml}$, observado durante o período chuvoso no transecto 2 , a um máximo de $6,5 \mathrm{mg} / \mathrm{ml}$ durante o período seco no transecto 1 (Fig. 2A e 2B). 


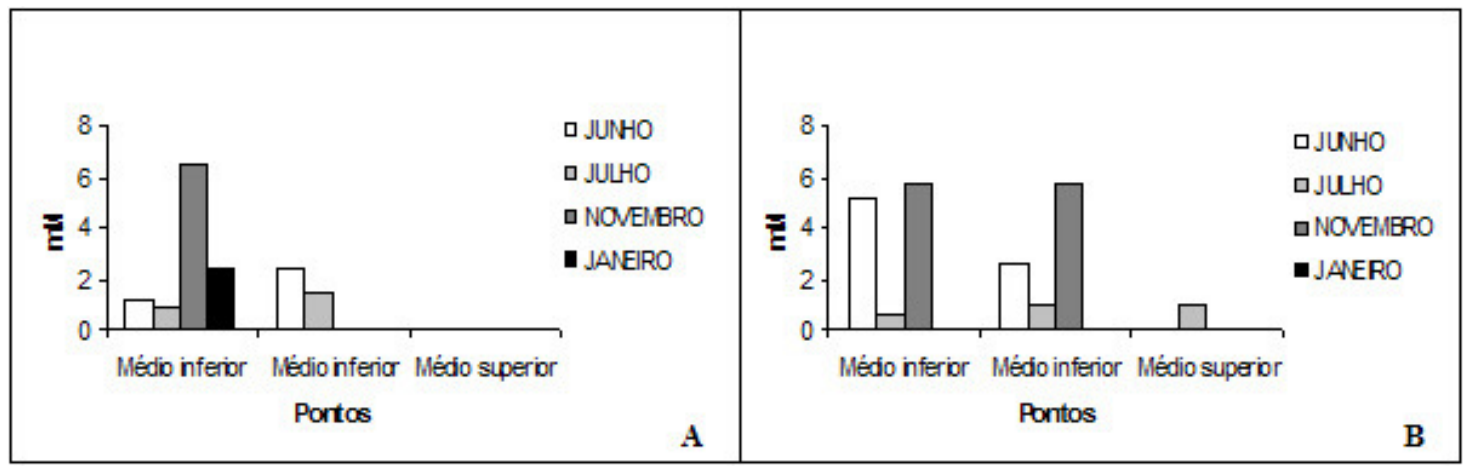

Figura 2 - Teores de oxigênio dissolvido na água intersticial no médio-litoral da margem sul da ilha de Itamaracá no período estudado ( $\mathrm{A}=$ transecto $1 ; \mathrm{B}=$ transecto 2$)$.

A areia foi a fração predominante no sedimento, com média de $98,34 \%$ (D.P.=1,22) do conteúdo de cada amostra, sendo insignificante a proporção de silte e argila (média $=0,23 \%$, D.P $=0,27$ ). De um modo geral, o tamanho dos grãos foi menor no transecto 1 que no transecto 2 , com os mais grosseiros sendo encontrados nos andares mais inferiores da praia, próximos à linha d’água, diminuindo no sentido do médio-litoral superior. O tamanho médio do grão do sedimento não variou significativamente com a profundidade, com média de
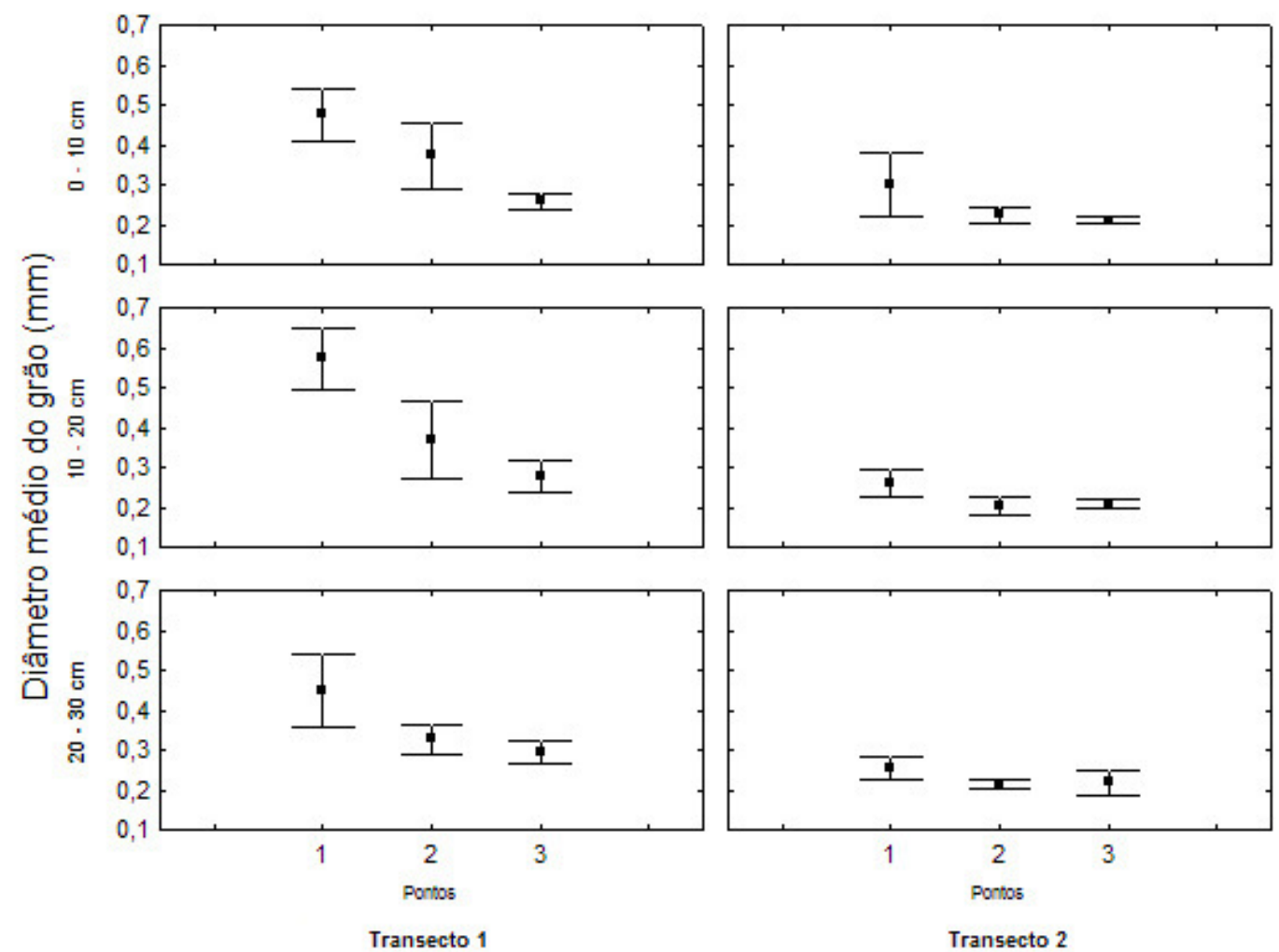

Figura 3 - Diâmetro médio dos grãos do sedimento no médio-litoral da margem sul da ilha de Itamaracá no período estudado (ponto $1=$ médio-litoral inferior, $2=$ médio-litoral, $3=$ médio-litoral superior). Barras verticais indicam desvio padrão. 

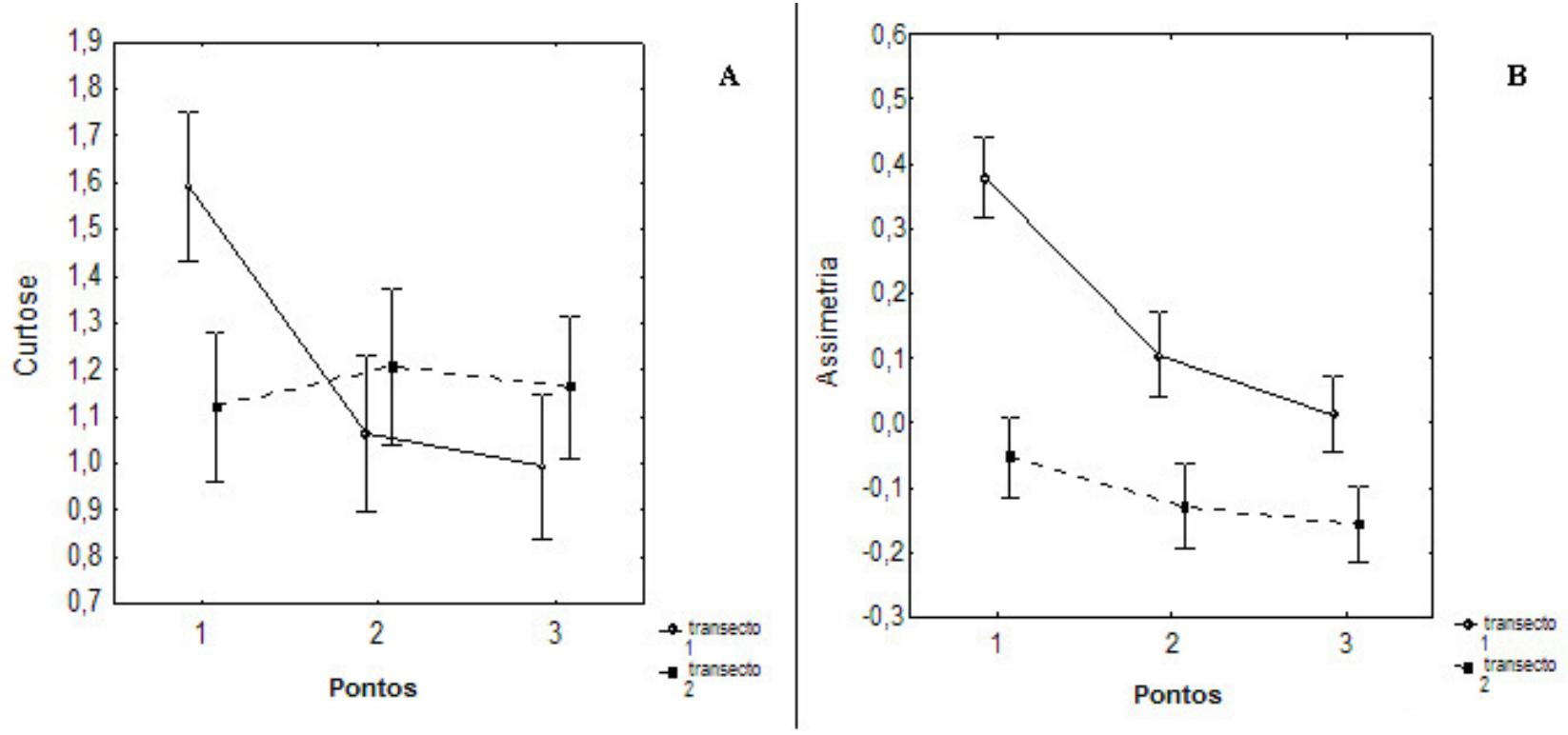

Figura 4 - Curtose (A) e assimetria (B) dos grãos do sedimento no médio-litoral da margem sul da ilha de Itamaracá no período estudado (ponto 1 = médio-litoral inferior, 2 = médio-litoral, 3 = médio-litoral superior). Barras verticais indicam intervalo de $95 \%$ de confiança.
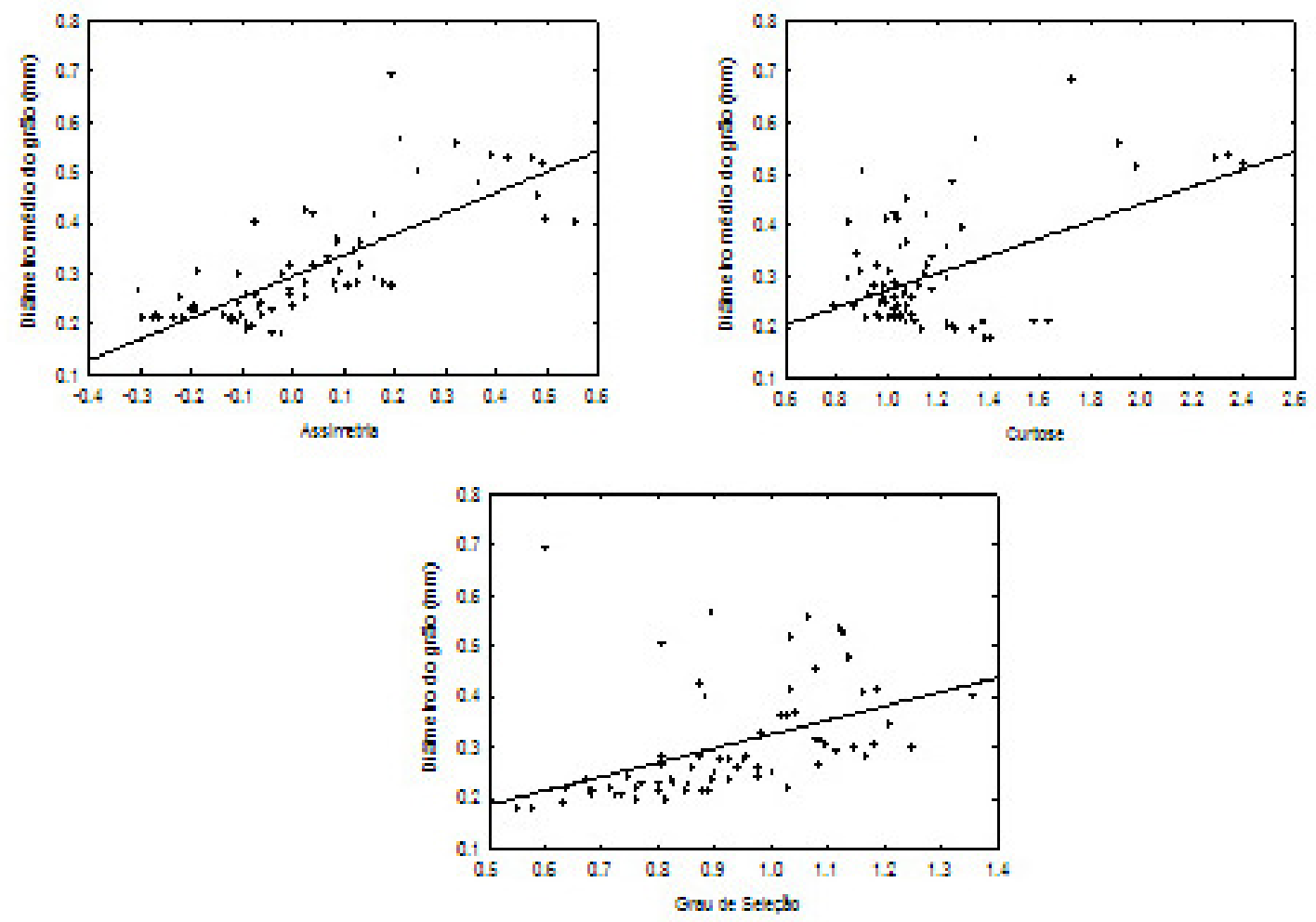

Figura 5 - Gráficos de dispersão do diâmetro médio do grão e: assimetria (A), curtose (B) e grau de seleção (C), do sedimento no médio-litoral da margem sul da ilha de Itamaracá no período estudado.

Foram encontrados 307 representantes de Stygarctus bradypus. A diferença entre os transectos, pontos, estratos e meses foi significativa (ANOVA fatorial $\left.F_{(12,144)}=1,973 ; p=0,031\right)$. $S$. bradypus ocorreu, praticamente, apenas na parte superior do médio-litoral, com 304 organismos, sendo 296 indivíduos encontrados no transecto 1 , com uma densidade média (em todo o transecto) de 4,21 ind. $10 \mathrm{~cm}^{-2}$. No andar intermediário estiveram presentes apenas durante o período seco, sendo a densidade média (considerando os dois 
transectos) de 0,03 ind. $10 \mathrm{~cm}^{-2}$. A espécie não foi encontrada no médio-litoral inferior (Fig. 6).
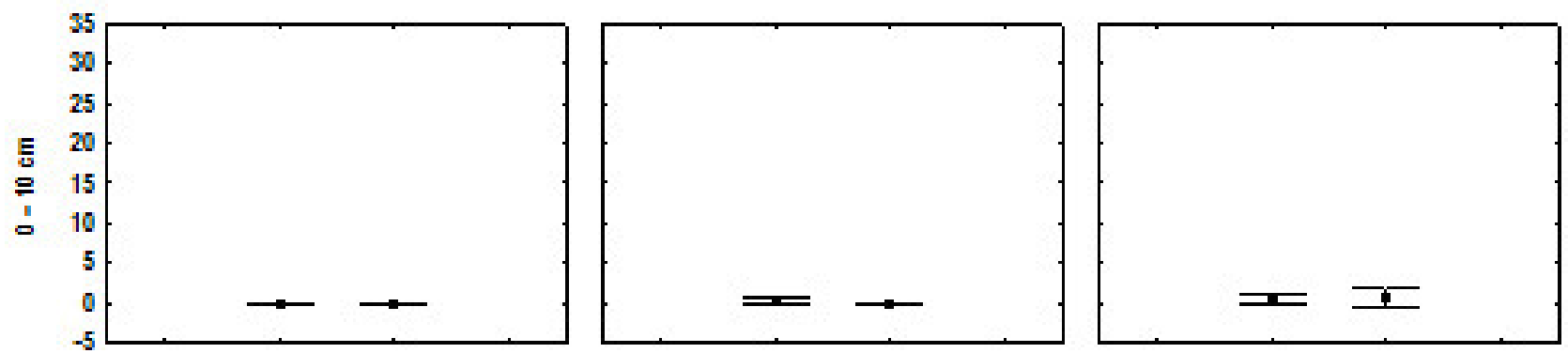

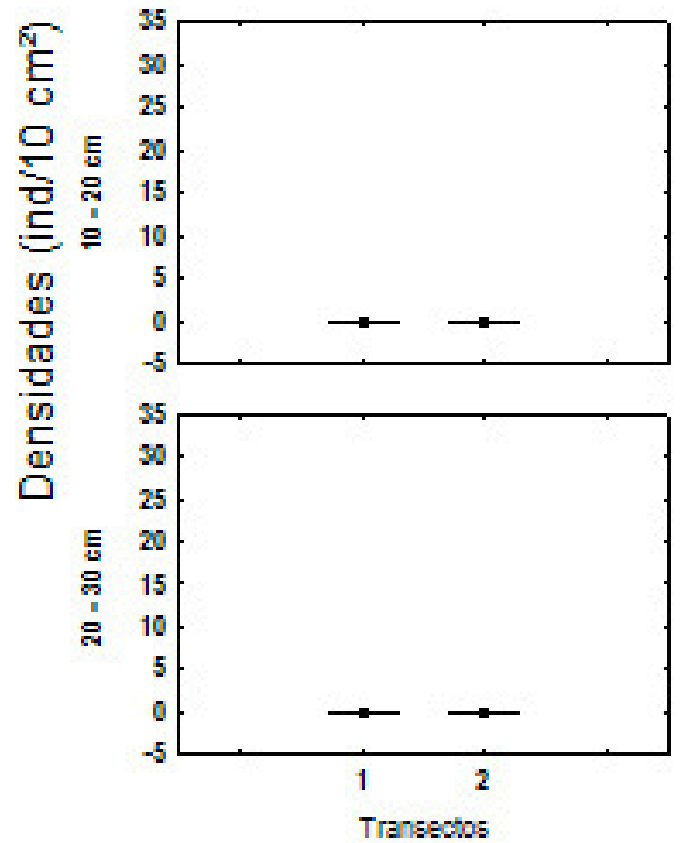

Médio Inferior
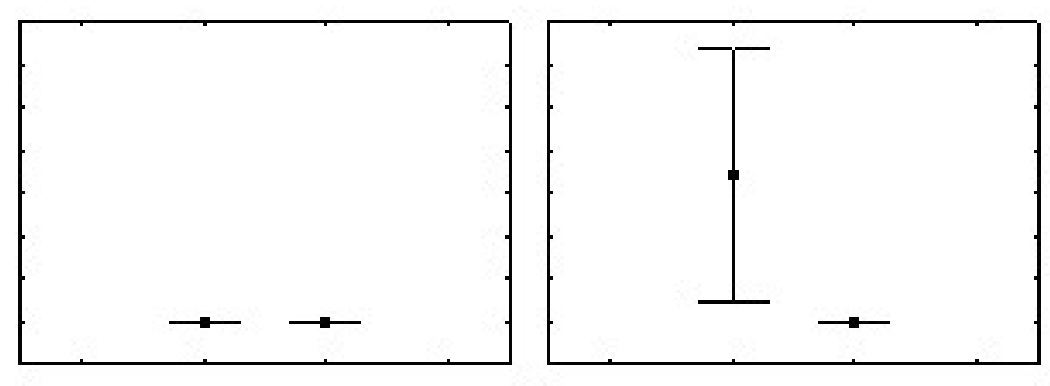

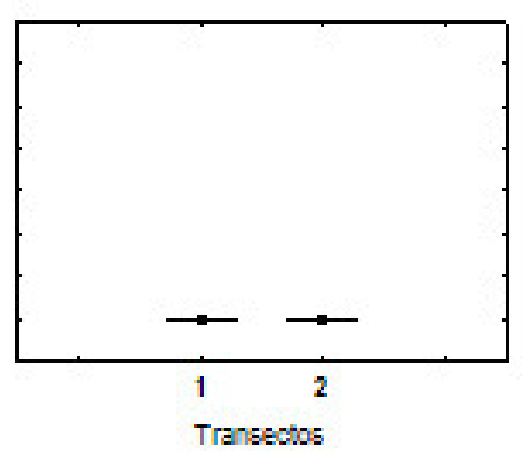

Médio-litoral

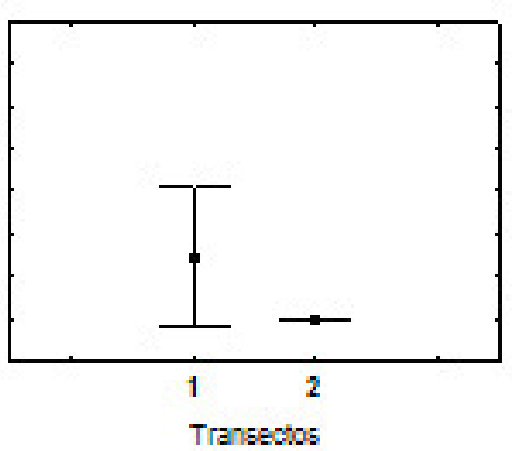

Médio Superior

Figura 6 - Densidades médias de Stygarctus bradypus na margem sul da ilha de Itamaracá no período estudado. Barras verticais indicam intervalo de $95 \%$ de confiança.

Com relação aos estratos sedimentares, $66,34 \%$ dos indivíduos foram encontrados entre 10 $\mathrm{cm}$ a $20 \mathrm{~cm}, 28,10 \%$ de $20 \mathrm{~cm}$ a $30 \mathrm{~cm}$ e apenas $5,56 \%$ de $0 \mathrm{~cm}$ a $10 \mathrm{~cm}$, sendo que nos estratos intermediário e profundo do médio-litoral superior no transecto $1, S$. bradypus foi significativamente mais abundante do que em todos os outros pontos estudados. $\mathrm{O}$ estrato intermediário foi significativamente maior nos meses de junho e novembro, e o estrato profundo, nos meses de julho e janeiro (Tukey, $p<0,0001$ ). Deste modo, analisando o conjunto de dados, não foram encontradas diferenças significativas entre as populações presentes nas amostras referentes aos períodos seco e chuvoso (ANOVA fatorial, $F_{(4,180)}=0,3346, p=0,85441$ ).

A análise de correlação aplicada aos dados de densidade de Stygarctus bradypus não indicou haver correlação significativa desta com nenhum dos parâmetros granulométricos aferidos. Por outro lado, foi detectada uma correlação negativa regular $e$ significativa entre as densidades de $S$. bradypus e a presença de água intersticial nos sedimentos $(R=-0,48$ e $p<0,05$ ). 


\section{DISCUSSÃO}

$\mathrm{Na}$ área de estudo, considera-se como principais agentes estruturadores do sedimento o vento e o hidrodinamismo (ondas e correntes), sendo o maior diâmetro dos grãos sedimentares restritos aos andares superiores da praia no transecto 1 uma característica das praias do litoral pernambucano (Mabesoone \& Coutinho 1970). Apesar de não termos obtido achados conclusivos a respeito da influência da presença da água intersticial sobre a espécie, os valores mais altos de oxigênio dissolvido encontrados no transecto 1 , juntamente com a presença de grãos mais grosseiros, sugerem maior circulação de água no local. Grimaldi De Zio et al. (1983a) relatam maiores densidades de $S$. bradypus em sedimentos dominados por areias médias como os encontrados neste estudo, apesar de Renaud-Mornant \& AnselmeMoizan (1969) afirmarem não haver relação entre o tamanho do grão e o tamanho dos indivíduos desta espécie.

$\mathrm{Na}$ deposição de grãos sedimentares nos andares superiores da praia pela ação das marés os tardígrados podem funcionar no sistema como partículas finas (Victor-Castro et al., 1999). A família Stygarctidae apresenta características morfológicas que a adaptam ao transporte passivo pelas correntes de marés, e por outro lado dificultam sua locomoção no meio intersticial (Grimaldi De Zio et al. 1983a; Ramazotti \& Maucci 1983). Renaud-Debyser \& Salvat (1963) referem importantes deslocamentos para Stygarctus provocados pela circulação de água nas mudanças de fases do ciclo de marés. No caso de $S$. bradypus pode ser que esteja atuando o mesmo mecanismo na margem sul da ilha de Itamaracá, sendo a população menos numerosa durante as marés baixas, e colonizando os estratos sedimentares mais inferiores como de hábito para a espécie. Bell \& Sherman (1980), Hagerman \& Rieger (1981) e Sibert (1981) corroboram as evidências de dispersão passiva na coluna d'água. Da Rocha et al. (2000) encontraram na mesma localidade do presente estudo populações muito mais expressivas de $B$. pennaki em amostragens realizadas durante 0 período de maré alta que nas da maré baixa, justificando o fato pelo transporte dos indivíduos pelas correntes de maré.
As maiores densidades de $S$. bradypus no estrato sedimentar de $10 \mathrm{~cm}$ a $20 \mathrm{~cm}$ de profundidade coincide com relatos sobre a sua bio-ecologia no Hemisfério Norte: Gaugler (2002) coletou 23 espécimes de $S$. bradypus em praia arenosa da Carolina do Sul, os quais estavam distribuídos de 30 $\mathrm{cm}$ a $60 \mathrm{~cm}$ de profundidade, resultado que coincide com a afirmação de Renaud-Mornant \& Pollock (1971), segundo os quais Stygarctus ocorre preferencialmente em maiores densidades de $40 \mathrm{~cm}$ a $60 \mathrm{~cm}$ de profundidade na areia. Kristensen \& Higgins (1984) encontraram Stygarctus gourbaultae em até $1,8 \mathrm{~m}$ de profundidade na Flórida (EUA). Pollock (1970) diz ainda que $S$. bradypus pode permanecer fundo na areia devido às menores variações de temperaturas nos estratos inferiores da coluna sedimentar.

Em Itamaracá provavelmente as variações das densidades populacionais entre o período seco e o período chuvoso não foram significativas devido ao fato de não haver mudanças drásticas de temperatura, sendo que diversos estudos sobre meiofauna associam as mudanças de densidades populacionais às variações sazonais (Heip et al. 1985). Em regiões tropicais os poucos trabalhos existentes associam as mudanças na estrutura da comunidade ao ciclo de chuvas (Pattnaik \& Rao, 1990; Ingole \& Parulekar, 1998). Da Rocha et al. (2004) em estudo realizado na mesma localidade observaram $27{ }^{\circ} \mathrm{C}$ no mês de julho e um máximo de $28,5{ }^{\circ} \mathrm{C}$ no mês de janeiro. Em regiões temperadas, onde as estações são mais demarcadas, são comuns baixas densidades no inverno, sendo explicadas pela alteração na temperatura ou diminuição do microfitobentos (Rudnick et al. 1985; Santos et al. 1996). Especificamente com relação a $S$. bradypus, Renaud-Mornant \& Anselme-Moizan (1969) relatam variações numéricas de caráter sazonal em praia francesa. Em estudo realizado em praia arenosa da Carolina do Norte (EUA), Lindgren (1971) encontrou poucos exemplares durante $\mathrm{O}$ inverno $\left(14{ }^{\circ} \mathrm{C}\right)$, entretanto as concentrações no verão $\left(28{ }^{\circ} \mathrm{C}\right)$ alcançaram densidades de no mínimo 15 indivíduos por $100 \mathrm{~cm}^{3}$ de sedimento.

Hulings \& Gray (1976) sugerem que em praias arenosas temperadas os fatores biológicos são importantes na estruturação das comunidades 
meiofaunísticas no verão, enquanto no inverno os fatores físico-químicos são mais relevantes. RenaudMornant \& Pollock (1971), por outro lado, relatam populações numerosas de $S$. bradypus em praias situadas perto de bancos de macrófitas. Segundo esses autores, sua distribuição é determinada pela presença ou ausência do alimento apropriado no sedimento. Estas plantas seriam arrancadas do seu substrato e lavadas em praias onde forneceriam restos vegetais para os tardigrados fitófagos, ou ainda transportá-los a outras localidades. Gerlach (1977) propôs que a dispersão de organismos meiofaunísticos pode se processar também através de objetos flutuantes, incluindo folhas e algas.

Portanto, o caráter fortemente pontual da distribuição de $S$. bradypus na margem sul da ilha de Itamaracá parece ser decorrente tanto da proximidade do banco de Halodule wrightii presente no infralitoral da margem leste da ilha, como do fato destes organismos, por seu tamanho reduzido, funcionarem no sistema como partículas finas, depositados nos andares superiores da praia pelas correntes de marés.

\section{REFERÊNCIAS}

BELL, SS \& KS SHERMAN. 1980. Tidal ressuspension as an mechanism for meiofauna dispersal. Mar. Ecol. Prog. Ser., 3: 245-249.

COBB, N. 1917. A. Note on nemas. Contrib. Sci. Nematol., 5: 117128.

DA ROCHA, CMC, V FONSÊCA-GENEVOIS \& FJ VICTORCASTRO. 2000. Distribuição espaço-temporal de Batillipes pennaki Marcus, 1946 (Tardigrada, Heterotardigrada) na margem sul da ilha de Itamaracá (Pernambuco - Brasil). Trop. Oceanogr., 28: 35-46.

DA ROCHA, CMC, FONSÊCA-GENEVOIS, V., FJ VICTORCASTRO, TNC BEZERRA, $V$ VENEKEY \& AP BOTELHO. 2004. Environmental interactions of Batillipes pennaki (Tardigrada, Heterotardigrada) in a tropical sandy beach (Itamaracá, Pernambuco, Brazil). Meiofauna Marina, 13: 79-86.

DE ZIO, S. 1965. Distribuzione del mesopsammon in rapporto alla distanza dalla línea di riva ed alla distanza da um corso d'acqua salmastra. Boll. Zool. 32 (2): 525-537.

DE ZIO GRIMALDI, S \& M GALLO D'ADDABBO. 2001. Further data on the Mediterranean Sea Tardigrade Fauna. Zool. Anz. 240: $345-360$.

DE ZIO GRIMALDI, S, M D'ADDABBO GALLO, R SANDULLI \& R D'ADDABBO. 2003. Checklist of the Italian marine Tardigrada. Meiofauna Marina. 12: 97-135.

DOS SANTOS, ECL, MM VERÇOSA, JRB SOUZA \& CMC DA ROCHA. 2006. Distribuição espacial do gênero Batillipes Richters, 1909 (Tardigrada, Heterotardigrada), na margem sul da ilha de Itamaracá, Pernambuco, Brasil. In: ALBUQUERQUE, U. P.; ASC VERAS, FJ FREIRE \& MAL JUNIOR. (eds.) Caminhos da Ciência. EDUFRPE, Recife, cap. 4: 63-77.

ELMGREN, R. 1966. Baltic benthos communities and the role of meiofauna. Contrib. Asko. Lab. Univ. of Stockholm. 14: 1-31.

FLEEGER, JW \& AW DECHO. 1987. Spatial variability of interstitial meiofauna: a review. Stygologia, 3 (1): 35-54.

FINDLAY, SEG. 1981. Small scale spatial distribution of meiofauna on a mud and sand flat. Estuar. Coast. Shelf Sci., 12: 471-484.

FOLK, L. \& C WARD. 1957. Brazos River bar: a study in the significance of grain size parameters. J. Sedim. Petrology. 27 (1): 3-27.

GAUGLER, MS. Marine Interstitial Tardigrades and Other Meiofauna of Huntington Beach, South Carolina. 2002. 148p. Dissertação (Mestrado em Ciências Biológicas) - East Tennessee State University, 2002.

GERLACH, AS. 1977. Means of meiofauna dispersal. Mikrofauna Meeresboden, 61: 89-103.

GRIMALDI DE ZIO, S., M D'ADDABBO GALLO \& MR MORONE DE LUCIA. 1982a. Neostygarctus acanthophorus n. gen. n. sp.: Nuovo tardigrado marino del Mediterraneo. Cah. Bio. Mar., 23: 319-323.

GRIMALDI DE ZIO, S., M D'ADDABBO GALLO \& MR MORONE DE LUCIA. 1983b. Marine Tardigrades of the "Secca dell'Armelia" (Ionian Sea), and redescription of Raiarctus colurus Renaud-Mornant 1981 (Heterotardigrada). Oebalia, 9: 33-42.

GRIMALDI DE ZIO, S., M D'ADDABBO GALLO, MR MORONE DE LUCIA \& P GRIMALDI. 1980a. Ulteriori dati sui Tardigradi del mesopsammon di alcune spiagge pugliesi. Thalassia Salent., 10: 45-65.

GRIMALDI DE ZIO, S. \& M D'ADDABBO GALLO. 1987. Archechiniscus minutus sp. nov. and its systematic position within Arthrotardigrada (Tardigrada: Heterotardigrada). In: Bertolani, R. (ed.), Biology of Tardigrades. Selected Symposia and Monographs U.Z.I., Mucchi - Moderna. p. 253-260.

GRIMALDI DE ZIO, S., RM DE LUCIA \& M D'ADDABBO GALLO. 1983a. Marine Tardigrades Ecology. Oebalia, 9: 15-31.

GRIMALDI DE ZIO, S., MR DE LUCIA, M D'ADDABBO GALLO \& P GRIMALDI. 1979. Osservazione su alcuni Tardigradi di una spiaggia pugliese e descrizione di Batillipes adriaticus sp. nov. (Heterotardigrada). Thalassia Salent., 9: 39-59.

GRIMALDI DE ZIO, S., MR MORONE DE LUCIA, M D'ADDABBO GALLO \& P GRIMALDI. 1980b. Conoscenze attuali sulla distribuzione dei Tardigradi marine nel Bacino del Mediterraneo. Mem. Biol. Mar. Oceanogr. 10 Suppl. 6:403-405.

HAGERMAN, GM \& RM RIEGER. 1981. Dispersal of benthic meiofauna by wave and current action in Bogue Sound, N.C. USA. Mar. Ecol., 2: 245-270.

HEIP, C, M VINCX \& G VRANKEN. 1985. The ecology of marine nematodes. Oceanogr. Mar. Biol. Annu. Rev., 23: 399-489.

HONDT, JL d'. 1970. Gastrotriches, Kinorhynques, Rotiféres, Tardigrades. Inventaire de la faune marine de Roscoff. Ed. Stat. Biol. Roscoff., 1-29.

HULINGS, NC \& JS GRAY. 1971. A manual for the study of meiofauna. Smithson. Contrib. Zool. Smithsonian Institution Press, Washington. $84 \mathrm{p}$. 
HULINGS, NC \& JS GRAY. 1976. Physical factors controlling abundance of meiofauna on tidal and atidal beaches. Mar. Biol., 34: 77-83.

INGOLE, PS \& AH PARULEKAR. 1998. Role of the salinity in structuring the intertidal meiofauna of a tropical estuarine beach: field evidences. Indian J. Mar. Sci., 27: 356-361.

KRISTENSEN, RM \& RP HIGGINS. 1984. A New Family of Arthrotardigrada (Tardigrada: Heterotardigrada) from the Atlantic Coast of Florida, U.S.A. Trans. Am. Microscopical Soc., 103 (3): 295-311.

KRISTENSEN, RM \& B NEHUAUS. 1999. The ultrastructure of the tardigrade cuticle with special attention to marine species. Zool. Anz., 238: 261-281.

LINDGREN, EW. 1971. Psammonlittoral marine tardigrades from North Carolina and their conformity to worldwide zonation patterns. Cah. Biol. Mar., 12: 481-96.

MABESOONE, JM. \& PN COUTINHO. 1970. Littoral and shallow marine geology of Northern and Northeastern Brazil. Trabalhos Oceanográficos, 12: 1-214.

MARTINS, MH DE A. Caracterização morfológica e vulnerabilidade do litoral da ilha de Itamaracá. Recife. 1997. 104 f. Tese (Mestrado em Geociências). Universidade Federal de Pernambuco, Brasil, 1997.

McGYNTY, M. \& R HIGGINS. 1968. Ontogenetic variation of taxonomic characters of two marine Tardigrades with the description of Batillipes bullacaudatus n. sp. Trans. Am. Microsc. Soc., 87: 252-262.

McLACHLAN, A. 1983. Sandy beach ecology - a review. In: MCLACHLAN, A \& T ERASMUS (eds.) Sandy beaches as ecosystems: 1st International Symposium on Sandy Beaches, Port Elizabeth, South Africa. Developments in Hydrobiology. Chap. 19: 321-380

MEDEIROS, C, B KJERFVE, M ARAÚJO \& S NEUMANN-LEITÃO. 2001. The Itamaracá Estuarine Ecosystem, Brazil. In: U. SEELINGER, \& B KJERFVE. (ed.) Coastal Marine Ecosystems of Latin America. Ecological Studies. Springer-Verlag. Heidelberg. 144: 71-81.

PATTNAIK, A \& MVL RAO. 1990. Composition and distribution of the interstitial meiofauna of the sandy beach at Golpapur South Orissa Coast. Indian J. Mar. Sci., 19: 165-170.

POLLOCK LW. 1970. Distribution and dynamics of interstitial Tardigrada at Woods Hole,Massachusetts, USA. Ophelia., 7: 145-66.

RAO, GC. \& PN GANAPATI. 1968. The interstitial fauna inhabiting the beach sands of Waltair coast. Proc. Nat. Inst. Sci. Índia, 34: 82-125.

RAMAZZOTTI, G. \& W MAUCCI. 1983. II phylum Tardigrada (III edizione riveduta e aggiornata). Mem. Ist. Ital. Idrobiol., 1012p.

RENAUD-DEBYSER, J. 1956. Repartition de deux tardigrades Batillipes mirus Richters et Stygarctus bradypus Schulz dans un segment de plage du Bassin d'Archon. C R Acad SC Paris, 243: 1365-1369.

RENAUD-DEBYSER, J. 1959a. Sur quelques tardigrades du Bassin d'Arcachon. Vie Millieu, 10 (2):135-146.
RENAUD-DEBYSER, J. 1959b. Études sur la faune interstitielle des Iles Bahamas. III. Tardigrades. Vie Millieu., 10 (3):296-302.

RENAUD-DEBYSER, J. 1963. Récherches ecologiques sur la faune interstitielle des sables. Bassin d'Arcachon île de Bimini, Bahamas. Vie Millieu., 15 Suppl.: 1-157.

RENAUD-DEBYSER, J. \& B SALVAT. 1963. Eléments de prospérité des biotopes des sediments meubles intertidaux et écologie de leurs populations en microfaune et macrofaune. Vie Millieu., 14 (3) : 463-550.

RENAUD-MORNANT, J \& MM ANSELME-MOIZAN. 1969. Stades larvaires du tardigrade marin Stygarctus bradypus Schulz et position systématique des Stygarctidae. Bull. Mus. Natn. Histoire Naturelle Paris., 41(4): 883-893.

RENAUD-MORNANT, J. \& LW POLLOCK. 1971. A review of the systematics and ecologyof marine Tardigrada. Smithson. Contrib. Zool., 76: 109-117.

RENAUD-MORNANT, J. \& P SERENE. 1967. Note sur la Microfaune de la cote orientale de la Malaisie. (Gastrotriches, Annellidess, Tardigrades et Copépodes Harpacticides). Cah. Pacif. 11:51-73.

RUDNICK, DT, R ELMGREN \& JB FRITHSEN. 1985. Meiofaunal prominente and benthic seasonality in a coastal marine ecosystem. Oecologia. 16: 157-170.

SANTOS, PJP, J CASTEL \& LP SOUZA-SANTOS. 1996. Seasonal variability of meiofauna abundace in the oligo-mesohaline area of the Gironde Estuary, France. Estuar. Coast. Shelf Sci., 43:549-563.

SCHMIDT, P. 1968. Die quantitative Verteilung und Populationsdynamik des Mesopsammons am GezeitenSandstrand der Nordseeinsel Sylt I. Faktorengefüge und biologische Gliederung des Lebensraumes. Int. Rev. ges. Hydrobiol., 58: 723-779.

SCHMIDT, P. 1969. Die quantitative Verteilung und Populationsdynamik des Mesopsammons am GezeitenSandstrand der Nordsee-Insel Sylt II. Quantitative Verteilung und Populationsdynamik einzelner Arten. Int. Rev. ges. Hydrobiol., 54: 95-174.

SCHULZ, E. 1951. Über Stygarctus bradypus n. g. n. sp., einen Tardigraden aus dem Küstengrundwasser, und seine phylogenetische Bedeutung. Kiel. Meeresforsch, 7 (1):86-97.

SIBERT, JR. 1981. Intertidal hiperbenthic population in the Nanaimo Estuary. Mar. Biol., 64: 259-265.

SOUZA-SANTOS, LP, PJP SANTOS \& V FONSÊCA-GENEVOIS. 2004. Meiofauna. In: ESKINAZI-LEÇA, E, S NEUMANNLEITÃO \& MF COSTA (eds.) Oceanografia: um cenário tropical. Editora Bagaço, pp. 529-554.

VICTOR-CASTRO, FJ, V FONSÊCA-GENEVOIS, L LIRA \& CMC DA ROCHA. 1999. Efeito da granulometria e da topografia sobre a distribuição de Batillipes pennaki Marcus, 1946 em zona tropical típica: restinga do Paiva, Pernambuco, Brasil. Trabalhos Oceanográficos, 27 (2): 89-102.

Recebido: 05/12/2007

Aceito: 09/09/2008 
